\title{
First report of MEFV duplication in FMF patient
}

\author{
G Sarrabay ${ }^{*}$ D Méchin, B Dumont, M André, I Touitou \\ From 8th International Congress of Familial Mediterranean Fever and Systemic Autoinflammatory Diseases \\ Dresden, Germany. 30 September - 3 October 2015
}

\section{Introduction}

Familial mediterranean fever (FMF) is a rare monogenic disease and the prototype of autoinflammatory disorders. It is caused by mutations in the $M E F V$ gene and is autosomal recessively inherited. Most mutations are missense substitutions, small deletions are quite rare, and only three nonsense mutation has been described (http://fmf.igh.cnrs. fr/ISSAID/infevers/). Large rearrangements have been searched for in the frame of a collaborative project including 216 patients but were not identified.

\section{Objectives}

We report here the first case of $M E F V$ duplication in a FMF patient.

\section{Patients and methods}

The proband is a 21 years-old woman who presented with classical FMF phenotype: recurrent fever, arthralgia, and abdominal pain with vomiting. Attacks lasted three days and biological inflammation was documented with elevated C-reactive protein. Her father is Armenian and her mother Malagasy, and both are asymptomatic. We performed Sanger analysis (ABI3130x, Life Technologies) of the $M E F V$ gene, quantitative polymerase chain reaction (qPCR) (LighCycler, Roche) and deep-sequencing (Nextera Rapid Capture, Illumina) (MiSeq, Illumina). Microsatellite analysis (ABI3130x, Life Technologies) was also performed.

\section{Results}

We identified a well-known severe mutation: p.Met694Val, and a controversial variant: p.Glu148Gln. Parental testing confirmed that the variants were non-allelic. Sanger sequencing displayed unbalanced ratio of the mutated and wild type alleles. Mosaicism was excluded because all polymorphisms were found at the same 1:2 ratio. DNA contamination was ruled out through microsatellite analysis. We thus suspected a gene micro-rearrangement. qPCR

CHU Montpellier, Montpellier, France 